\title{
Tunable Spin Wave Nonreciprocity in a Magnetic System With Two Rectangular Blocks
}

\section{Dongpyo Seo}

Gwangju Institute of Science and Technology (GIST)

\section{S. Hwang}

Gwangju Institute of Science and Technology (GIST)

\section{Byeongro Kim}

Gwangju Institute of Science and Technology (GIST)

\section{Yeonhee Yang}

Gwangju Institute of Science and Technology (GIST)

\section{Seungha Yoon}

Korea Institute of Industrial Technology

\section{B. K. Cho ( $\nabla$ chobk@gist.ac.kr)}

Gwangju Institute of Science and Technology (GIST)

\section{Research Article}

Keywords: Nonreciprocity, Tunable spin wave, magnetic system, two rectangular blocks, key properties

Posted Date: August 11th, 2021

DOI: https://doi.org/10.21203/rs.3.rs-786203/v1

License: (c) (i) This work is licensed under a Creative Commons Attribution 4.0 International License.

Read Full License 


\title{
Tunable spin wave nonreciprocity in a magnetic system with two rectangular blocks
}

\author{
Dongpyo Seo ${ }^{1}$, S. Hwang ${ }^{1}$, Byeongro Kim ${ }^{1}$, Yeonhee Yang ${ }^{1}$, Seungha Yoon ${ }^{2}$, B. K. Cho ${ }^{1 *}$ \\ ${ }^{1}$ School of Materials Science and Engineering, Gwangju Institute of Science and Technology \\ (GIST), Gwangju, 61005, Republic of Korea \\ ${ }^{2}$ Green Energy \& Nano Technology R\&D Group, Korea Institute of Industrial Technology \\ (KITECH), Gwangju, 61012, Republic of Korea
}

*Email: chobk@gist.ac.kr, Tel.: +82-62-715-2318, Fax: +82-62-715-2304

Nonreciprocity and propagation of spin waves are key properties to develop spin-based electronics, such as magnetic memory, spin information and logic devices. To date, a nonreciprocity ratio cannot be manipulated in a system, while large values are achieved because of the geometrical magnetic configuration. In this study, we suggest a new magnetic system with two blocks, in which the nonreciprocity ratio can be changed between 0.276 and 1.43 by adjusting the excitation frequency between $7.8 \mathrm{GHz}$ and $9.4 \mathrm{GHz}$. Because the two blocks have different widths, they have their own spin wave excitation frequency ranges. Indeed, the spin wave intensities in the two blocks, detected by the Brillouin light scattering spectrum, were observed to be frequency-dependent, yielding tunable nonreciprocity. Thus, this study provides a new path to enhance the application of spin waves in spin-based electronics. 


\section{Introduction}

There has been great interest in spin-based electronics because of its high potential for application to new electronic devices, such as signal processing and data storage. One of the data storage devices is magnetic random access memory (MRAM), which manipulates spin states using spin transfer torque $e^{1-3}$ or spin orbit torque ${ }^{4-6}$. In signal transfer and processing applications, magnonics, which use spin waves as information carriers, have attracted considerable attention. Due to the absence of charge carrier transportation, spin wave devices have some advantages such as low power consumption and high processing speed ${ }^{7}$. In addition, many studies have proposed magnonic switches, transistors, and logic devices in recent years ${ }^{8-12}$.

One of the interesting properties of spin waves in device applications is the nonreciprocal propagation of spin waves. The magnetostatic surface spin wave (MSSW), which moves coplanar with and perpendicular to the magnetized direction, propagates with directional dependent intensity due to the asymmetry of the induced field. Such characteristics are called spin wave nonreciprocity. Thus, spin wave nonreciprocity can provide additional degrees of freedom for the control of signal propagation ${ }^{12,13}$. Spin wave nonreciprocity has large potential for applications in switches and logic devices due to selectively unidirectional spin wave propagation ${ }^{12}$, while in devices that require bidirectional signal propagation, the spin wave intensity should be the same in both directions ${ }^{14}$.

Spin wave nonreciprocity is quantified by comparing the spin wave intensities propagating in different directions. Manipulation of the intensity ratio, defined as the nonreciprocity ratio, is the main target in recent studies ${ }^{14-16}$. Various parameters have been utilized to control the spin wave nonreciprocity: the frequency of microwaves, magnitude of the external field, 
excitation antenna width and film thickness ${ }^{14,17}$. The nonreciprocity ratio values were reported to be small $(0.4 \sim 0.7)$. Moreover, the wave propagation direction with a larger amplitude could not be controlled. To overcome these problems, Kwon et al. studied a bilayer of tantalum and permalloy and found that it gives a large value of nonreciprocity $(\sim 60)$ and a preferred direction of spin propagation ${ }^{16}$. Deorani et al. also suggested a system with two antennas on both sides of a permalloy film, and obtained a nonreciprocity ratio larger than $50^{18}$. However, magnetic film systems have no flexibility to change the nonreciprocity ratio and the wave propagation direction once the system is fabricated. It would be of great interest to find a way to control the nonreciprocity ratios and direction in a system.

Here, we suggest two bar-type permalloy $\left(\mathrm{Ni}_{80} \mathrm{Fe}_{20}\right)$ blocks that have different widths and are located on a line with a narrow separation $(\sim 0.3 \mu \mathrm{m})$. Because the two blocks have their own frequency range for spin excitation, the excitation characteristics would have a significant dependence on the RF frequency. Thus it is found that both the spin wave nonreciprocity ratio and propagation direction can be controlled by the applied excitation RF current.

\section{Experimental details}

Spin wave propagation was detected using a Brillouin light scattering (BLS) spectroscopy system. The BLS spectroscopy is an optical tool that uses inelastic scattering of photons with some quasiparticles, similar to Raman spectroscopy, working between photons and phonons. The BLS spectroscopy has the advantage of investigating excitations in the $\mathrm{GHz}$ frequency regions where, in general, spin waves prevail. A schematic diagram of the BLS measurement setup is shown in Fig. 1(a). To obtain a high-resolution image of spin wave propagation, a 


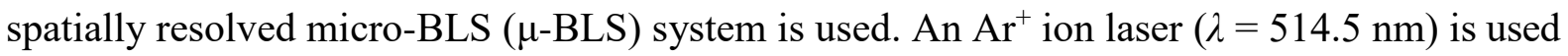
as the monochromatic light source. The scattered light from the sample was transferred to a Fabry-Perot interferometer with two etalons. High contrast of the signal is obtained as the light is transferred through both etalons. The spatial interval of the measurement is set to 100 and $200 \mathrm{~nm}$ for the $x$ - and $y$-directions, respectively. The sample surface and laser spot were visualized by a CCD camera for real-time measurement observation.

SEM images of samples A and B are shown in Fig. 1(b) and 1(c), respectively. Sample A consists of two rectangular blocks one with a width of $1.61 \mu \mathrm{m}$ and the other with a width of $3.14 \mu \mathrm{m}$, which have a gap of $0.3 \mu \mathrm{m}$ between them. Sample B consists of two identical rectangular blocks with a width of $3.09 \mu \mathrm{m}$ and the same gap. The blocks are multilayers of $\mathrm{Ta}(3 \mathrm{~nm}) / \mathrm{Ni}_{80} \mathrm{Fe}_{20}(20 \mathrm{~nm}) / \mathrm{SiO}_{2}(5 \mathrm{~nm})$. The $\mathrm{RF}$ microwave current antenna covers the gap across the two blocks, as shown in Fig. 1. The antenna is a stack of $\mathrm{SiO}_{2}(40 \mathrm{~nm}) / \mathrm{Ta}(3$ $\mathrm{nm}) / \mathrm{Cu}(150 \mathrm{~nm}) / \mathrm{Ta}(5 \mathrm{~nm})$ layers. Blocks and antennas are patterned by an e-beam lithography process.

A spin wave is generated with an applied field of $H_{x}=800$ Oe along the $x$-direction. An RF microwave current with a power of $P=100 \mathrm{~mW}$ flows through the antenna in the same direction as the external field.

\section{Results and discussion}

The dispersion relation is calculated by considering both dipole-dipole and exchange interactions ${ }^{19,20}$. The spin wave frequency of mode number $n$ on a thin film can be expressed $\operatorname{as}^{20}$ 


$$
\omega_{n}^{2}=\left(\omega_{H}^{n}+\alpha \omega_{M} k^{2}\right)\left[\omega_{H}^{n}+\alpha \omega_{M} k^{2}+\omega_{M} F(k L)\right]
$$

where $\alpha$ is the exchange constant, $k^{2}=k_{n x}^{2}+k_{y}^{2}$ is an in-plane component of the wave vector, $\omega_{H}^{n}=\omega_{H}+\omega_{M} N_{x}(x), \omega_{H}=\gamma H_{e x}, \omega_{M}=4 \pi \gamma M_{S}, \gamma$ is the gyromagnetic ratio, $M_{S}$ is the saturation magnetization, and $H_{e x}$ is an external field. The demagnetization factor $N_{x}$ is

$$
N_{x}=\frac{1}{\pi}\left[\arctan \frac{t}{2 x+w}-\arctan \frac{t}{2 x-w}\right]
$$

where $t$ is the thickness and $w$ is the width of the film. In equation (1), $F(k L)$, which represents a quantized matrix element of the dipole-dipole interaction can be written as

$$
F(k L)=1+P(k L)[1-P(k L)]\left(\frac{\omega_{M}}{\omega_{H}^{n}+\alpha \omega_{M} k^{2}}\right) \times\left(\frac{k_{y}^{2}}{k^{2}}\right)-P(k L)\left(\frac{k_{n x}^{2}}{k^{2}}\right)
$$

where $P(k L)$ is

$$
P(k L)=1-\frac{1-\exp (k L)}{k L}
$$

The exchange constant and gyromagnetic ratio of the permalloy used in this calculation are $\alpha=2.7975 \times 10^{-17} \mathrm{~m}^{2}$ and $\frac{\gamma}{2 \pi}=2.8 \times 10^{-3} \mathrm{GHz} / \mathrm{Oe}$, respectively. The measured saturation magnetization is 816 Oe.

The calculated dispersion relations of the spin wave with mode number $n=1$ for the blocks are shown in Fig. 2(a). It shows that the dispersion has a clear dependence on the block width, i.e., the upper dispersion curve (in red) for the block with a width of $3.14 \mu \mathrm{m}$ and the lower curve (in black) for that with a width of $1.61 \mu \mathrm{m}$. Thus, the ferromagnetic resonance frequency in the wider block is found to be $8.06 \mathrm{GHz}$, and that for the narrower block is $7.80 \mathrm{GHz}$. It is known that the spin wave wavelength should be larger than the width of the microwave antenna for efficient excitation ${ }^{15}$. Considering the $1.9 \mu \mathrm{m}$ width antenna 
used in this study, a spin wave with a wavevector larger than $3.31 \mu \mathrm{m}^{-1}$ would not be excited. As a result, the spin wave frequency, which is allowed in the narrower block, is found in a range of $7.80 \leq \omega \leq 9.15 \mathrm{GHz}$ (denoted by a black arrow in Fig. 2(a)). The allowed frequency for the wider block is also found in a range of $8.06 \leq \omega \leq 9.51 \mathrm{GHz}$ (denoted by a red arrow).

The measured BLS intensities for the two blocks are plotted in terms of the frequency in Fig. 2(b). It is clear that the BLS intensity is strong in the frequency ranges for each block and decreases rapidly outside the ranges. Thus, nonreciprocity is expected near both ends of the frequency ranges. If the RF frequency is larger than $9.15 \mathrm{GHz}$, the spin wave in the wider block is excited while the spin wave in the narrower block is negligible. The opposite situation occurs if the RF frequency is smaller than $8.06 \mathrm{GHz}$. This means that the spin waves in the field ranges of $7.80 \mathrm{GHz} \leq \omega \leq 8.06 \mathrm{GHz}$ and $9.15 \mathrm{GHz} \leq \omega \leq 9.51 \mathrm{GHz}$ are excited only in the narrower and wider blocks, respectively. The waves propagate in a specific direction, i.e., upward in the narrower block and downward in the wider block. On the other hand, in a frequency range of $8.06 \leq \omega \leq 9.15 \mathrm{GHz}$, the spin waves are excited in both blocks at the same time and propagate along both directions. This implies that frequency modulation can be a valid way to manipulate the spin wave nonreciprocity and direction in this geometry.

To observe spin wave propagation, spatial profiles of the spin waves in sample A with various frequencies are observed and plotted in Fig. 3. At a frequency of $7.6 \mathrm{GHz}$, no spin wave excitation is observed except for the spin wave of the edge mode in the wider block (Fig. 3(a)). At the frequencies of $7.8 \mathrm{GHz}$ and $7.9 \mathrm{GHz}$, the spin waves are observed to be excited only in the narrower block (Figs. 3(b) and (c)). As the frequency increases (Figs. 3(d) and (e)), the spin waves in the wider block, as well as in the narrower block, start to be excited. At a frequency of $8.8 \mathrm{GHz}$ (Fig. 3(f)), the BLS spectrum clearly shows the spin wave 
excitation in both blocks with almost the same intensity. With a further increase in frequency (Figs. 3(g), (h) and (i)), the BLS intensity in the narrower block decreases while the intensity in the wider block remains strong. The observations in Fig. 3 are consistent with the discussion on spin wave excitation based on the dispersion relation. Asymmetric spin wave excitations are clearly observed depending on the frequency. This means that in the two block system (sample A), the spin wave nonreciprocity and propagation direction are manipulated by frequency variation.

From the data in Fig. 3, the spin wave intensity ratio between the narrower and wider blocks, defined as the nonreciprocity ratio, is plotted in Fig. 4. The data, indicated by red square symbols, represent the spin wave excitation in the narrower block and wave propagation upward. The data represented by black circle symbols, are for the excitation in both blocks and bidirectional propagation, and those by blue triangles are for the excitation in the wider block and propagation downward. The nonreciprocity ratio change from 0.276 to 1.43 in a system (sample $\mathrm{A}$ in this study), and the wave propagation direction also changes with the variation of frequency.

For comparison, spatial profiles of spin wave excitation in the two blocks with the same dimension (sample B) are plotted in Fig. 5. At low frequencies of $7.6 \mathrm{GHz}$ and $7.9 \mathrm{GHz}$, no excitation of the main mode wave, other than the edge mode, is observed in either the upper or lower blocks (Figs. 5(a) and (b)). At the frequencies of $8.4 \mathrm{GHz}, 8.8 \mathrm{GHz}$ and $9.4 \mathrm{GHz}$ (Figs. 5(c), (d) and (e)), the spin excitation in both blocks is observed with slight intensity differences. The intensity difference is due to nonsymmetric field application and a general feature of MSSW. Thus, the characteristics in sample B cannot be utilized for the control of nonreciprocity and propagation direction, as reported in previous research ${ }^{17}$. 


\section{Conclusion}

We investigated the spin wave characteristics in a magnetic system of two blocks, which have different width dimensions $(1.61$ and $3.14 \mu \mathrm{m})$ and are separated with a small gap $(0.3$ $\mu \mathrm{m})$. We found that the spin wave excitation and its propagation can be controlled by adjusting the excitation frequency. The spatial BLS spectrum shows that the spin wave is excited only in the narrower and wider blocks at low $(\approx 7.9 \mathrm{GHz})$ and high frequencies $(\approx$ $9.4 \mathrm{GHz})$, respectively, and in both blocks at frequencies $(\approx 8.8 \mathrm{GHz})$ between them. As a consequence, the spin wave propagation direction is determined to be unidirectional downward and upward at low and high frequencies, respectively, and bidirectional at the frequencies between them. These observations are found to be consistent with the analytical dispersion relations for the two blocks. The nonreciprocity ratio, defined as the BLS intensity ratio of the spin wave in the blocks, is determined to be in the range between 0.26 and 1.43 , depending on the excitation frequency.

The two-block system shows a new function of tunable nonreciprocity and propagation without structural modification. Compared to the pre-existing system, the discovery in this study affords an additional degree of freedom in signal processing. Our system will provide a new possibility for the applications of spin-based electronics, such as spin logic devices, switch devices, and spin wave-based data transporting systems. 


\section{References}

1. J. A. Katine, F. J. Albert, R. A. Buhrman, E. B. Myers, and D. C. Ralph, Phys. Rev. Lett., 84, 3149 (2000)

2. Y. Huai, F. Albert, P. Nguyen, M. Pakala, and T. Valet, Appl. Phys. Lett., 84, 3118 (2004)

3. K. L. Wang, J. G. Alzate and P. K. Amiri, J. Phys. D: Appl. Phys., 46, 074003 (2013)

4. S. Zhang, Phys. Rev. Lett., 85, 393 (2000)

5. I. M. Miron, K. Garello, G. Gaudin, P. Zermatten, M. V. Costache, S. Auffret, S. Bandierra, B. Rodmacq, A. Schuhl, and P. Gambardella, Nature, 476, 189 (2011)

6. L. Liu, O. J. Lee, T. J. Gudmundsen, D. C. Ralph and R. A. Buhrman, Phys. Rev. Lett., 109, 096602 (2012)

7. V. V. Kruglyak, S. O. Demokritov, and D. Grundler, J. Phys. D: Appl. Phys., 43, $264001(2010)$

8. A. V. Chumak, A. A. Serga, and B. Hillebrands, Nature Communications, 5, 4700 (2014)

9. R. Cheng, M. W. Daniels, J. Zhu, and D. Xiao, Scientific Reports, 6, 24223 (2016)

10. M. Balinskiy, H. Chiang, and A. Khitun, AIP Advances, 8, 056628 (2018)

11. T. Schneider, A. A. Serga, B. Leven, and B. Hillebrands, Appl. Phys. Lett., 92, 022505 (2008)

12. M. Jamali, J. H. Kwon, S. Seo, K. Lee, and H. Yang, Scientific Reports, 3, 3160 (2013)

13. P. Deorani, J. H. Kwon, and H. Yang, Current Applied Physics, 14, S129 (2014)

14. A. V. Sadovnikov, E. N. Beginin, S. E. Sheshukova, Yu. P. Sharaevskii, A. I. Stognij, N. N. Novitski, V. K. Sakharov, Yu. V. Khivintsev, and S. A. Nikitov, Phys. Rev. B., 99, 054424 (2019) 
15. K. Shibata, K. Kasahara, K. Nakayama, V. V. Kruglyak, M. M. Aziz, and T. Manago, J. Appl. Phys., 124, 243901 (2018)

16. M. Nakayama, K. Yamanoi, S. Kasai, S. Mitani, and T. Manago, Jpn. J. Appl. Phys., 54, $083002(2015)$

17. J. H. Kwon, J. Yoon, P. Deorani, J. M. Lee, J. Sinha, K. Lee, M. Hayashi, and H. Yang, Sci. Adv., 2, e1501892 (2016)

18. V. E. Demidov, M. P. Kostylev, K. Rott, P. Krzysteczko, G. Reiss, and S. O. Demokritov, Appl. Phys. Lett., 95, 112509 (2009)

19. R. Stamps, and R. Camley Solid State Physics: Volume 65, Cambridge, Massachusetts: Academic Press (2014)

20. B. A. Kalinikos and A. N. Slavin, J. Phys. C: Solid State Phys., 19, 7013 (1986)

21. K. Y. Guslienko, R. W. Chantrell, and A. N. Slavin, Phys. Rev. B, 68, 024422 (2003) 

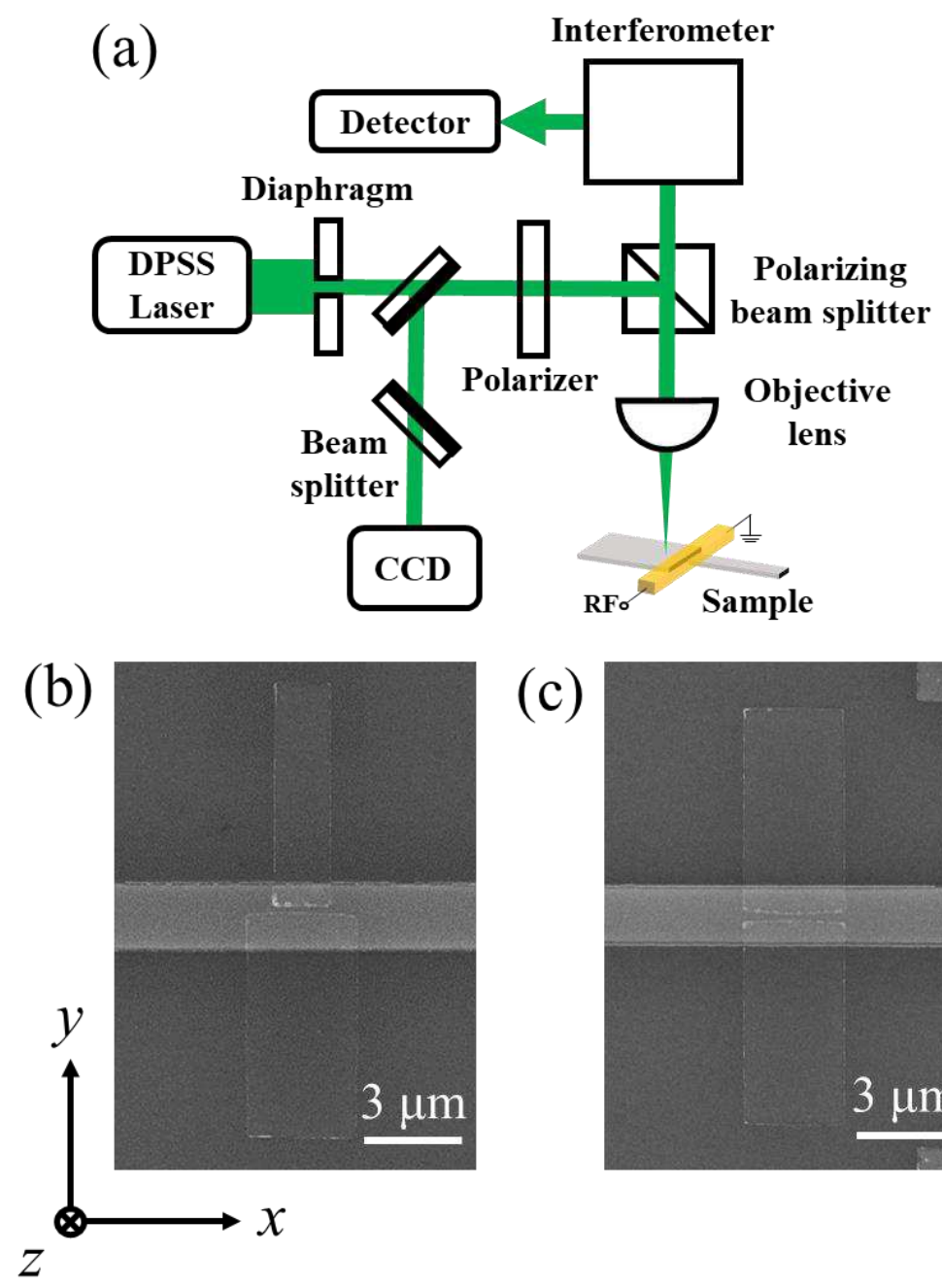

(c)

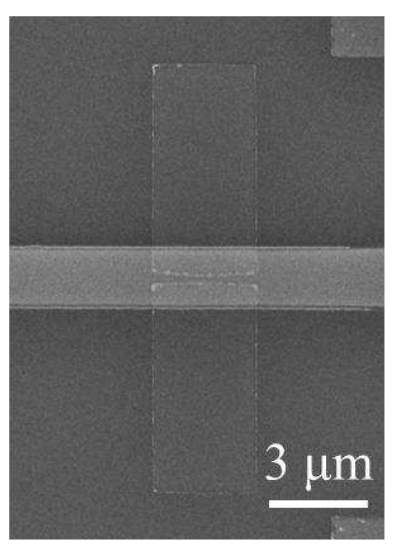

Figure 1. (a) Schematic diagram of the BLS measurement system. Scanning electron microscopy image of the magnetic blocks, separated by small gap and excitation antenna: (b) The two blocks have different widths. (c) The two blocks have identical width. 

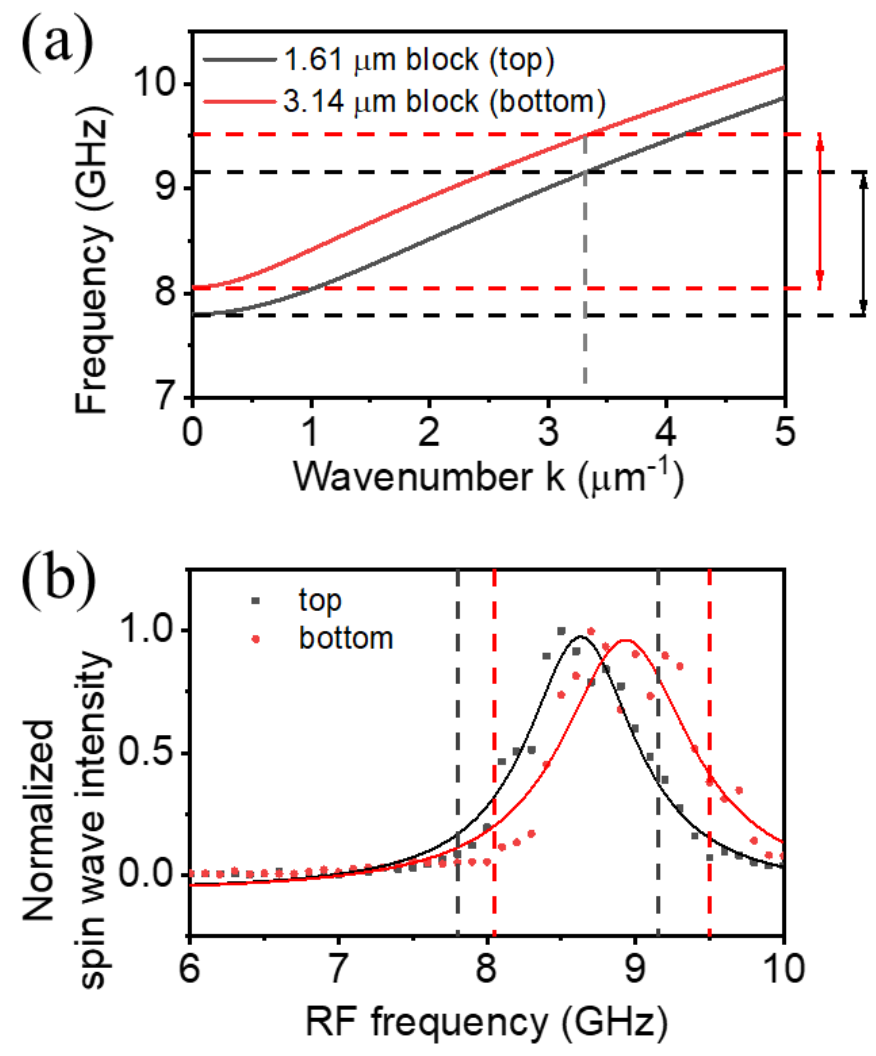

Figure 2. (a) Dispersion relation of the spin wave with mode number $n=1$ for NiFe blocks $1.61 \mu \mathrm{m}$ wide (black line) and $3.14 \mu \mathrm{m}$ wide (red line). (b) Normalized spin wave intensity of the two NiFe blocks in terms of the excitation RF frequency. 


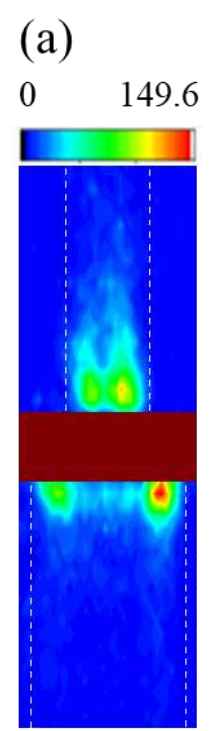

(f) (b)

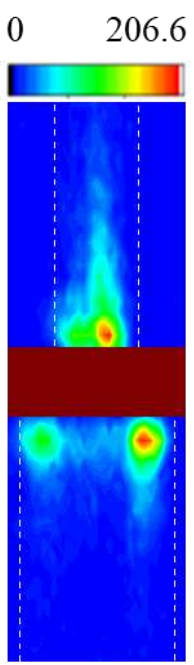

(g) (c)

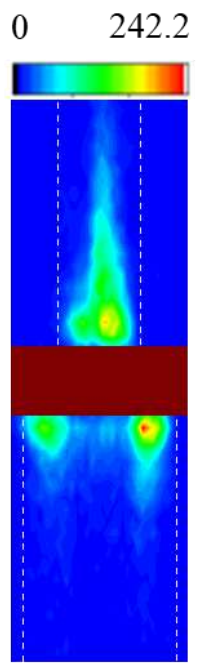

(h) (d) $\quad(\mathrm{e})$

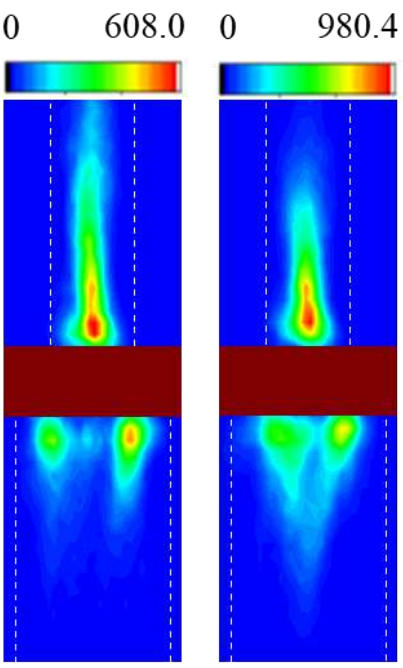

(i)

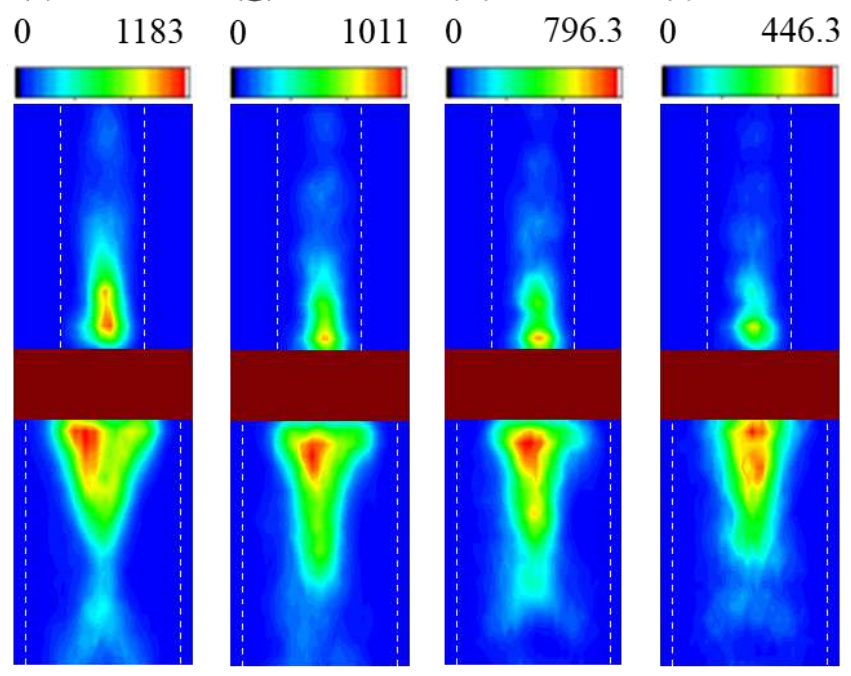

Figure 3. Spatial profiles of Brillouin light spectrum with various RF frequencies in the two blocks: (a) $7.6 \mathrm{GHz}$ (b) $7.8 \mathrm{GHz}$ (c) $7.9 \mathrm{GHz}$ (d) $8.2 \mathrm{GHz}$ (e) $8.4 \mathrm{GHz}$ (f) $8.8 \mathrm{GHz}$ (g) 9.0 GHz (h) $9.2 \mathrm{GHz}$ and (i) $9.4 \mathrm{GHz}$ 


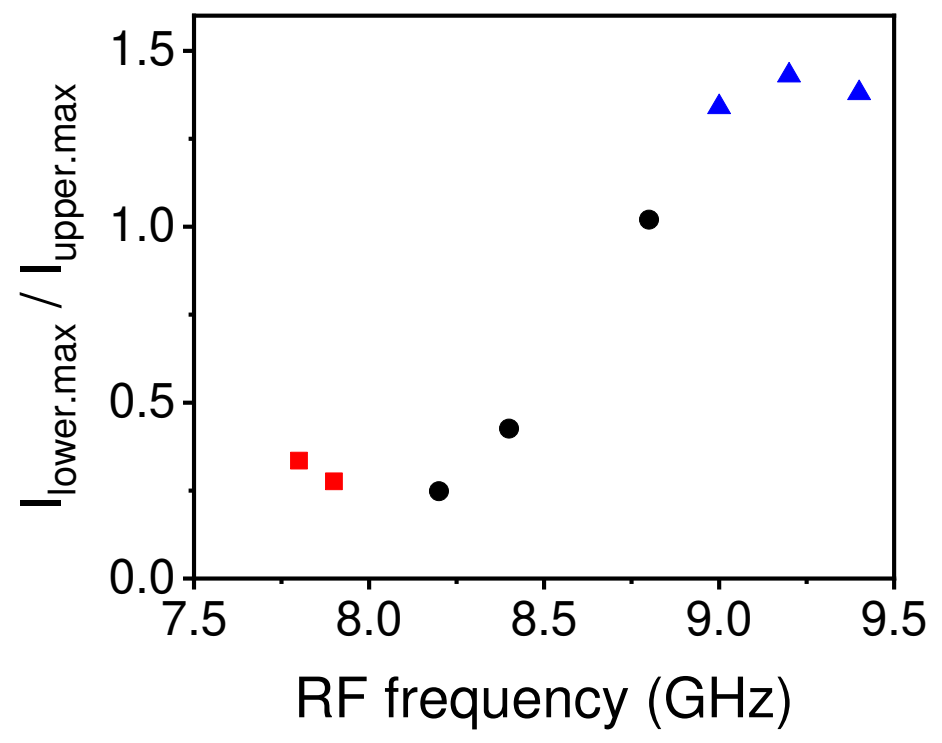

Figure 4. Intensity ratio (lower.max/lupper.max) of the Brillouin light scattering spectrum in Fig. 3, where lower.max and lupper.max are the maximum intensities in the wider and narrower blocks, respectively. Red squares represent spin wave excitation only in the narrower block, blue triangles excitation only in the wider block and black circles excitation in both blocks. 


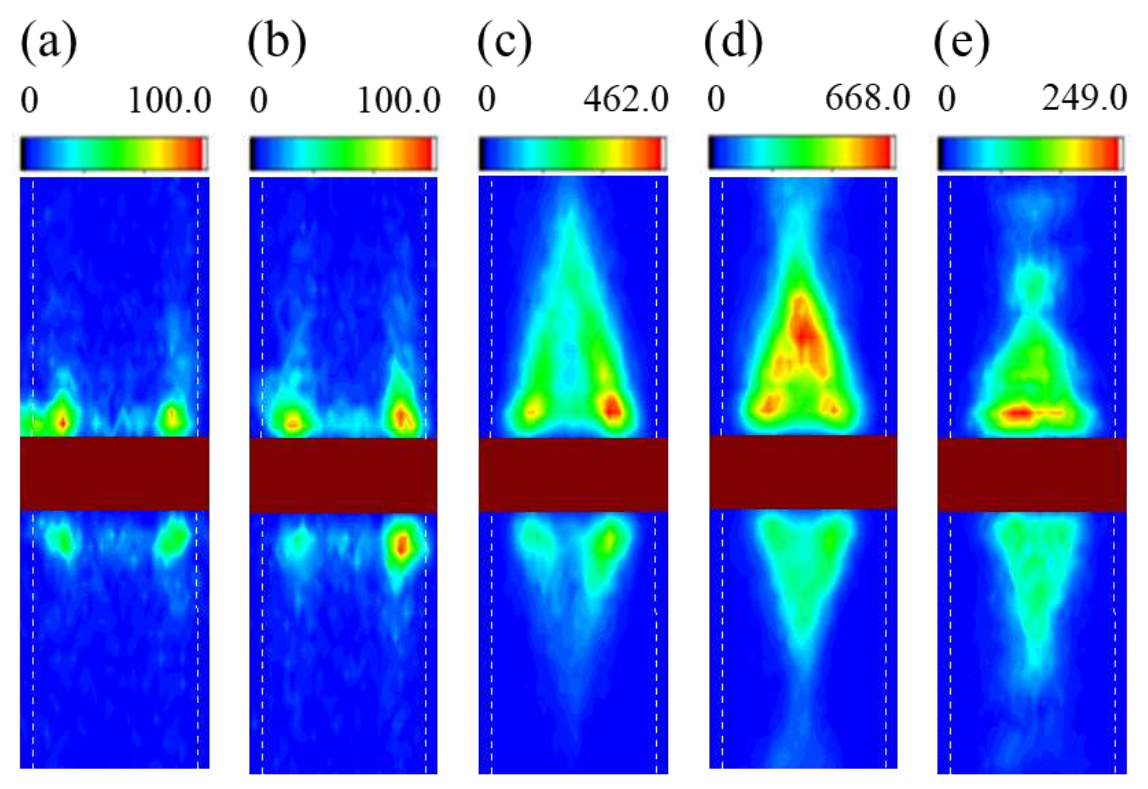

Figure 5. Spatial profiles of Brillouin light scattering spectrum with various RF frequencies in the two blocks with identical dimensions: (a) $7.6 \mathrm{GHz}$ (b) $7.9 \mathrm{GHz}$ (c) $8.4 \mathrm{GHz}$ (d) $8.8 \mathrm{GHz}$ and (e) $9.4 \mathrm{GHz}$ 


\section{Author contributions}

B.K.C. and D.S. conceived the project idea and planned the experiment. D.S. and S.H. fabricated samples and performed the analytical calculation. D.S. and B.R.K. performed the measurement. D.S., S.H., Y.Y., S.Y., and B.K.C. analyzed the data. B.K.C. led the work and wrote the manuscript with D.S. All authors discussed the results and commented on the manuscript.

\section{Acknowledgements}

This work was supported by GIST Research Institute (GRI) grant funded by the GIST in 2021, by the Korea Institute of Industrial Technology (KITECH), and by National Research Foundation of Korea (NRF) grant funded by the Ministry of Science and ICT (No. NRF2017R1A2B2008538). 